\title{
Enhancing Visualizations of Business Processes
}

\author{
Philip Effinger, Michael Kaufmann, and Martin Siebenhaller \\ Universität Tübingen, WSI für Informatik, Sand 13, 72076 Tübingen, Germany \\ \{effinger, mk, siebenha\}@informatik.uni-tuebingen.de
}

In today's business world, there exist multitudinous tools to model processes. For an automatic layout algorithm for those processes, it is important to take into account criteria like process flow and the semantic of the modeling notation. Most of the existing tools use standard layout approaches that often produce unsatisfying results since the calculated drawings are too dense and/or too large. This makes it difficult for users to understand the underlying process model.

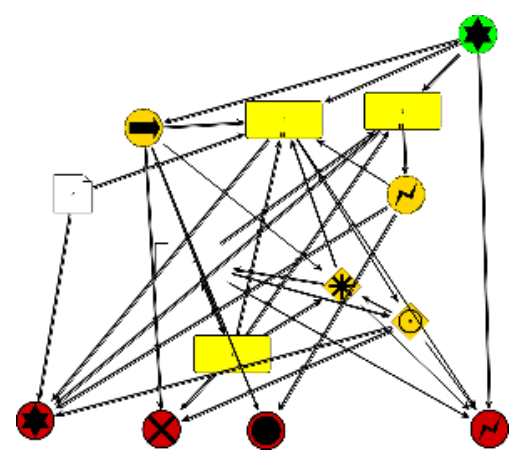

(a) BPMN-graph

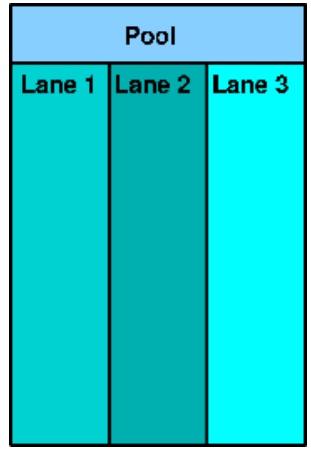

(b) Swimlanes/Pools

Fig. 1. (a) shows a graph representing a business process. It was drawn by starting with placing nodes and finally inserting straight-line edges. The different categories of BPMN elements [3] are represented by graph objects differing in color and shape.

The popular business process modeling notation (BPMN) [3] consists of the following categories of elements (see Fig. 1): Flow objects control the flow in a process and connecting objects are used to connect flow objects. Swimlanes/Pools partition flow objects into logical units, e.g. departments of a company. Annotations offer the possibility to add comments to flow and connecting objects.

For the core layout we use the orthogonal layout approach described in [2] that incorporates different constraints needed for the automatic layout of activity diagrams which are related to business process diagrams. The supported constraints include partitions, clusters as well as a common flow direction of edges which is especially important for such diagrams. To improve the layout quality we introduce two concepts - connectors and cuts.

Connectors replace edges by a pair of connector nodes, which are connected to the corresponding endpoints of the replaced edge (see Fig. 2). Connector nodes belonging 


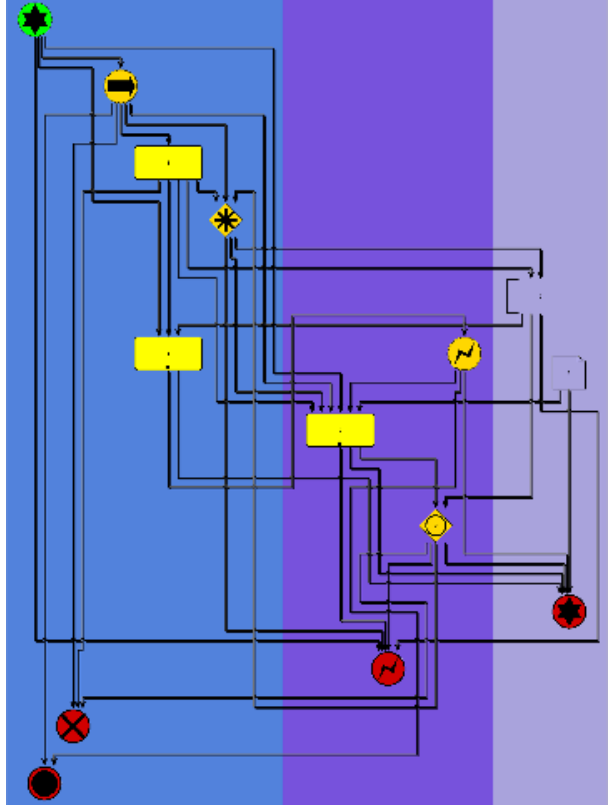

(a)

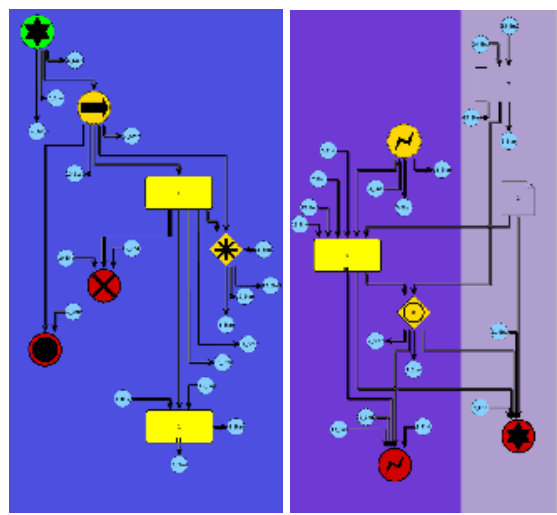

(b)

Fig. 2. Applying our approach to the BPMN-graph of Fig. 1. (a) shows the result after applying the core layout (the assignment of nodes to swimlanes is given as input). After inserting connectors and performing a vertical cut we obtain the sub-layouts shown in (b). Note, that the sum of the area of the resulting subgraphs is considerably smaller and the significant layout properties (embedding, shape and orientation) are maintained.

to the same edge get the same label to denote their correlation. Connectors offer a way to reduce the number of unaesthetic edges, i.e. edges with many bends and crossings. We determine candidates for a replacement by connectors by means of a badness function. In cases where process models become very complex and cannot easily be overlooked, it is desirable to split the resulting diagram into smaller pieces. The main objective for a cut is to split as few edges as possible and to obtain subgraphs of nearly equal size. We use the dual graph to find an appropriate route for a cut. The resulting subgraphs are relayouted using the sketch-driven approach described in [1]. It reduces the area of the drawing without changing the user's mental map (see Fig. 2).

\section{References}

1. Brandes, U., Eiglsperger, M., Kaufmann, M., Wagner, D.: Sketch-driven orthogonal graph drawing. In: Goodrich, M.T., Kobourov, S.G. (eds.) GD 2002. LNCS, vol. 2528, pp. 1-11. Springer, Heidelberg (2002)

2. Siebenhaller, M., Kaufmann, M.: Drawing activity diagrams. Technical Report WSI-2006-02, Wilhelm-Schickard-Institute, University of Tübingen (2006)

3. White, S.A.: Introduction to bpmn (2004), http: / / www . bpmn . org 